\title{
Analytical Investigation of Magnetohydrodynamic Flow over a Nonlinear Porous Stretching Sheet
}

\author{
Fazle Mabood ${ }^{1}$ and Nopparat Pochai ${ }^{2}$ \\ ${ }^{1}$ Department of Mathematics, University of Peshawar, Peshawar 25120, Pakistan \\ ${ }^{2}$ Department of Mathematics, King Mongkut's Institute of Technology Ladkrabang, Bangkok 10520, Thailand \\ Correspondence should be addressed to Nopparat Pochai; nop_math@yahoo.com
}

Received 30 March 2016; Revised 11 May 2016; Accepted 29 May 2016

Academic Editor: Giorgio Kaniadakis

Copyright (c) 2016 F. Mabood and N. Pochai. This is an open access article distributed under the Creative Commons Attribution License, which permits unrestricted use, distribution, and reproduction in any medium, provided the original work is properly cited.

We investigated the magnetohydrodynamic (MHD) boundary layer flow over a nonlinear porous stretching sheet with the help of semianalytical method known as optimal homotopy asymptotic method (OHAM). The effects of different parameters on fluid flow are investigated and discussed. The obtained results are compared with numerical Runge-Kutta-Fehlberg fourth-fifth-order method. It is found that the OHAM solution agrees well with numerical as well as published data for different assigned values of parameters; this thus indicates the feasibility of the proposed method (OHAM).

\section{Introduction}

Nonlinear differential equations are frequently arising from mathematical modeling of many physical phenomena. Several are solved by means of numerical methods and some are solved using the analytic methods such as perturbation $[1,2]$.

Researchers and engineers have paid more attention towards the analytical solution of boundary layer equations arising in numerous fluids phenomena [3-5]. The study of boundary layer flow for an incompressible fluid has many important applications in science and engineering, for example, the cooling of metallic plate in a cooling bath, the boundary layer along liquid film condensation process, and polymer industries.

In recent years, the analysis of magnetohydrodynamics (MHD) flow of a fluid over a stretching sheet has more popularity industrially and consequently becomes a fundamental problem in fluid dynamics [6-11]. McCormack and Crane [12] have initiated the stretching problem. The steady flow over a stretching sheet has numerous aspects, such as MHD flow, Non-Newtonian fluids, porous plate, porous medium, and heat transfer phenomena. Sakiadis $[13,14]$ is pioneer in this area who has investigated the boundary layer flow with uniform speed over continuously stretching surface. Later on, the work of Sakiadis was investigated and verified experimentally with different aspects by many researchers (see [15-17] and the references therein).

Most attention has so far been devoted to the analysis of flow of viscoelastic fluids [18-21] and the joint effect of viscoelasticity and magnetic field has been worked out by Ariel [22]. Khan et al. [23] studied MHD nonlinear porous stretching sheet using homotopy perturbation transform method (HPTM). Moreover, Chiam [24], Dandapat and Gupta [20], and Pavlov [25] have considered the motion of micropolar, power-law fluids and MHD flow over a stretching wall, respectively.

The optimal homotopy asymptotic method is a powerful approximate analytical technique that is straightforward to use and does not require the existence of any small or large parameter. Optimal homotopy asymptotic method (OHAM) is employed to construct the series solution of the problem. This method is a consistent analytical tool and it has already been applied to nonlinear differential equations arising in the science and engineering [26-28]. So far as we know there has been no OHAM solution of MHD flow over a nonlinear porous stretching sheet.

This paper is organized as follows. First in Section 2, we formulate the problem. In Section 3 we present basic principles of OHAM. The OHAM solution for MHD flow 
problem is given in Section 4. In Section 5, we analyze the comparison of the solutions using OHAM with numerical method (NM). Section 6 is devoted for the concluding remarks.

\section{Governing Equation}

We consider the MHD flow of an incompressible viscous fluid over a nonlinear porous stretching sheet at $y=0$. Electrically conducting fluid under the influence of applied magnetic field $B(x)$ normal to the stretching sheet, the induced magnetic field is assumed to be negligible. Under such assumption the MHD boundary layer equations are governed by

$$
\begin{aligned}
\frac{\partial u}{\partial x}+\frac{\partial v}{\partial y} & =0 \\
u \frac{\partial u}{\partial x}+v \frac{\partial u}{\partial y} & =v \frac{\partial^{2} u}{\partial y^{2}}-\sigma \frac{B^{2}(x)}{\rho} u
\end{aligned}
$$

where $u$ and $v$ are the velocity components in the $x$ - and $y$-directions, respectively. $\nu$ is the kinematic viscosity, $\rho$ is fluid density, $\sigma$ is the electrical conductivity, and $B(x)$ is the magnetic field strength, where $B(x)=B_{0} x^{(n-1) / 2}$.

The boundary conditions to the nonlinear porous stretching sheet are given below:

$$
\begin{gathered}
u(x, 0)=c x^{n}, \\
v(x, 0)=-V_{0}, \\
u(x, \infty)=0,
\end{gathered}
$$

where $c$ is the stretching parameter and $V_{0}$ is the porosity of the plate (whereas $V_{0}>0$ represents suction and $V_{0}<0$ corresponds to injection).

By introducing dimensionless variables for nondimensionalized form of momentum and energy equations,

$$
\begin{aligned}
& \eta=\sqrt{\frac{c(n+1)}{2 v}} x^{(n-1) / 2} y, \\
& u=c x^{n} f^{\prime}(\eta), \\
& v=-\sqrt{\frac{c v(n+1)}{2}} x^{(n-1) / 2}\left\{f(\eta)+\frac{n-1}{n+1} \eta f^{\prime}(\eta)\right\} .
\end{aligned}
$$

Using (3), the governing equations can be reduced to nonlinear differential equation, where $f$ is a function of the similarity variable $\eta$ :

$$
f^{\prime \prime \prime}+f f^{\prime \prime}-\beta f^{\prime 2}-M f^{\prime}=0,
$$

subject to the boundary conditions:

$$
\begin{aligned}
& f \longrightarrow K, \\
& f^{\prime} \longrightarrow 1 \\
& \quad \text { as } \eta \longrightarrow 0, \\
& f^{\prime} \longrightarrow 0 \quad \text { as } \eta \longrightarrow \infty,
\end{aligned}
$$

as

$$
\begin{aligned}
\beta & =\frac{2 n}{n+1}, \\
M & =\frac{2 \sigma \beta_{0}^{2}}{\rho c(1+n)}, \\
K & =\frac{V_{o}}{\sqrt{v c(n+1) / 2} x^{(n-1) / 2}} .
\end{aligned}
$$

$\beta$ is the nondimensional parameter, $M$ is the magnetic parameter, and $K$ is wall mass transfer parameter.

\section{Basic Principles of OHAM}

We review the basic principles of OHAM as developed in [26] in the following steps.

(i) Let us consider the following differential equation:

$$
A[v(x)]+a(x)=0, \quad x \in \Omega,
$$

where $\Omega$ is problem domain, $A(v)=L(v)+N(v)$, where $L$, $N$ are linear and nonlinear operators, $v(x)$ is an unknown function, and $a(x)$ is a known function.

(ii) Construct an optimal homotopy equation as

$$
\begin{aligned}
(1-q) & {[L(\phi(x ; q))+a(x)] } \\
- & H(q)[A(\phi(x ; q))+a(x)]=0,
\end{aligned}
$$

where $0 \leq q \leq 1$ is an embedding parameter and $H(q)=$ $\sum_{k=1}^{m} q^{k} C_{k}$ is auxiliary function on which the convergence of the solution is greatly dependent. The auxiliary function $H(q)$ also adjusts the convergence domain and controls the convergence region.

(iii) Expand $\phi\left(x ; q, C_{j}\right)$ in Taylor's series about $q$; one has an approximate solution:

$$
\begin{aligned}
& \phi\left(x ; q, C_{j}\right)=v_{0}(x)+\sum_{k=1}^{\infty} v_{k}\left(x, C_{j}\right) q^{k}, \\
& j=1,2,3, \ldots .
\end{aligned}
$$

Many researchers have observed that the convergence of the series equation (9) depends upon $C_{j}(j=1,2, \ldots, m)$; if it is convergent then we obtain

$$
\widetilde{v}=v_{0}(x)+\sum_{k=1}^{m} v_{k}\left(x ; C_{j}\right) .
$$

(iv) Substituting (10) into (7), we have the following residual:

$$
R\left(x ; C_{j}\right)=L\left(\widetilde{v}\left(x ; C_{j}\right)\right)+a(x)+N\left(\widetilde{v}\left(x ; C_{j}\right)\right) .
$$

If $R\left(x ; C_{j}\right)=0$, then $\widetilde{v}$ will be the exact solution. For nonlinear problems, generally this will not be the case. For determining $C_{j}(j=1,2, \ldots, m)$, Galerkin's Method, or the method of least squares, can be used.

(v) Finally, substitute these constants in (10) and one can get the approximate solution. 
TABLE 1: Comparison of OHAM results with numerical method for $K=0, \beta=5$, and $M=5$.

\begin{tabular}{|c|c|c|c|c|c|c|}
\hline \multirow{2}{*}{$\eta$} & \multicolumn{3}{|c|}{$f(\eta)$} & \multicolumn{3}{|c|}{$f^{\prime}(\eta)$} \\
\hline & OHAM & $\mathrm{NM}$ & $\%$ error & OHAM & NM & $\%$ error \\
\hline 0.0 & 0 & 0 & 0 & 1 & 1 & 0 \\
\hline 0.1 & 0.086665 & 0.086668 & 0.0034 & 0.746974 & 0.747032 & 0.0078 \\
\hline 0.2 & 0.151657 & 0.151663 & 0.0039 & 0.562191 & 0.562169 & 0.0039 \\
\hline 0.3 & 0.200655 & 0.200658 & 0.0014 & 0.424101 & 0.424023 & 0.0183 \\
\hline 0.4 & 0.237614 & 0.237598 & 0.0067 & 0.319327 & 0.319201 & 0.0394 \\
\hline 0.5 & 0.265301 & 0.265277 & 0.0090 & 0.237681 & 0.237665 & 0.0067 \\
\hline 0.6 & 0.285709 & 0.285688 & 0.0037 & 0.172819 & 0.172901 & 0.0474 \\
\hline 0.7 & 0.300262 & 0.300252 & 0.0033 & 0.119889 & 0.120035 & 0.1216 \\
\hline 0.8 & 0.309964 & 0.309969 & 0.0016 & 0.075254 & 0.075405 & 0.2002 \\
\hline 0.9 & 0.315493 & 0.315512 & 0.0060 & 0.036055 & 0.036162 & 0.2958 \\
\hline 1.0 & 0.317278 & 0.317303 & 0.0078 & 0 & 0 & 0 \\
\hline
\end{tabular}

\section{Series Solution via OHAM}

According to the OHAM, applying (8) to (4),

$$
\begin{aligned}
& (1-q)\left(f^{\prime \prime}+f^{\prime}\right) \\
& \quad-H(q)\left(f^{\prime \prime \prime}+f f^{\prime \prime}-\beta f^{\prime 2}-M f^{\prime}\right)=0,
\end{aligned}
$$

where primes denote differentiation with respect to $\eta$.

We consider $f$ and $H(q)$ as the following:

$$
\begin{aligned}
f & =f_{0}+q f_{1}+q^{2} f_{2}, \\
H(q) & =q C_{1}+q^{2} C_{2} .
\end{aligned}
$$

Using (13) in (12) and some simplifying and rearranging the terms based on the powers of $q$, we obtain zeroth-, first-, and second-order problems as follows.

Zeroth Order Problem. Consider

$$
f_{0}^{\prime \prime}(\eta)+f_{0}^{\prime}(\eta)=0
$$

with boundary conditions

$$
\begin{aligned}
& f_{0}(0)=K, \\
& f_{0}^{\prime}(0)=1 .
\end{aligned}
$$

Its solution is

$$
f_{0}(\eta)=K+1-e^{-\eta}
$$

First Order Problem. Consider

$$
\begin{aligned}
f_{1}^{\prime \prime}+ & f_{1}^{\prime} \\
& -C_{1}\left(e^{-\eta}-M e^{-\eta}+\left(K+1-e^{-\eta}\right) e^{-\eta}-\beta e^{-2 \eta}\right) \\
= & 0,
\end{aligned}
$$

with boundary conditions

$$
\begin{aligned}
& f_{0}(0)=0, \\
& f_{0}^{\prime}(0)=0 .
\end{aligned}
$$

It solution is

$$
\begin{aligned}
f_{1}\left(\eta, C_{1}\right)= & \frac{1}{2} C_{1} e^{-2 \eta}+C_{1} M(1+\eta) e^{-\eta} \\
& +C_{1} K(1+\eta) e^{-\eta}+\frac{1}{2} C_{1} \beta e^{-2 \eta} \\
& -C_{1}(1+\beta) e^{-\eta} \\
& +\frac{C_{1}}{2}(1-2 M-2 K+1) .
\end{aligned}
$$

And this goes on.

We obtain the three-term solution using OHAM for $q=$ $1:$

$$
\tilde{f}\left(\eta, C_{1}, C_{2}\right)=f_{0}(\eta)+f_{1}\left(\eta, C_{1}\right)+f_{2}\left(\eta, C_{1}, C_{2}\right) .
$$

We use the method of least squares to obtain the unknown convergent constants $C_{1}, C_{2}$ in (20); for particular case, if $M=1, K=0$, and $\beta=1.5$, then the values of $C_{1}, C_{2}$ are $C_{1}=-0.2233887095 ; C_{2}=0.8569476324$.

\section{Results and Discussion}

Table 1 shows the comparison of OHAM results with numerical (NM) for different values of parameters; in Table 2 we compare the numerical values of $f^{\prime \prime}(0)$ via OHAM with existing solution $[23,24]$. It is noteworthy to mention here that the OHAM gives lowest $\%$ error than other methods. This analysis shows that OHAM suits for MHD boundary layer flow problems. In Figures 1-3, we have shown the effects of the dimensionless parameter $\beta$, the magnetic parameter $M$, and the mass transfer parameter $K$ with various assigned values. 
TABLE 2: Comparison of $f^{\prime \prime}(0)$ via OHAM with other methods for various values of $M$ at $K=1$ and $\beta=1.5$.

\begin{tabular}{lcccccc}
\hline$M$ & Chiam [24] & HPTM [23] & Present results & $\begin{array}{c}\text { \% error Chiam } \\
{[24]}\end{array}$ & $\begin{array}{c}\text { \% error HPTM error } \\
{[23]}\end{array}$ & 0.2826 \\
present results
\end{tabular}

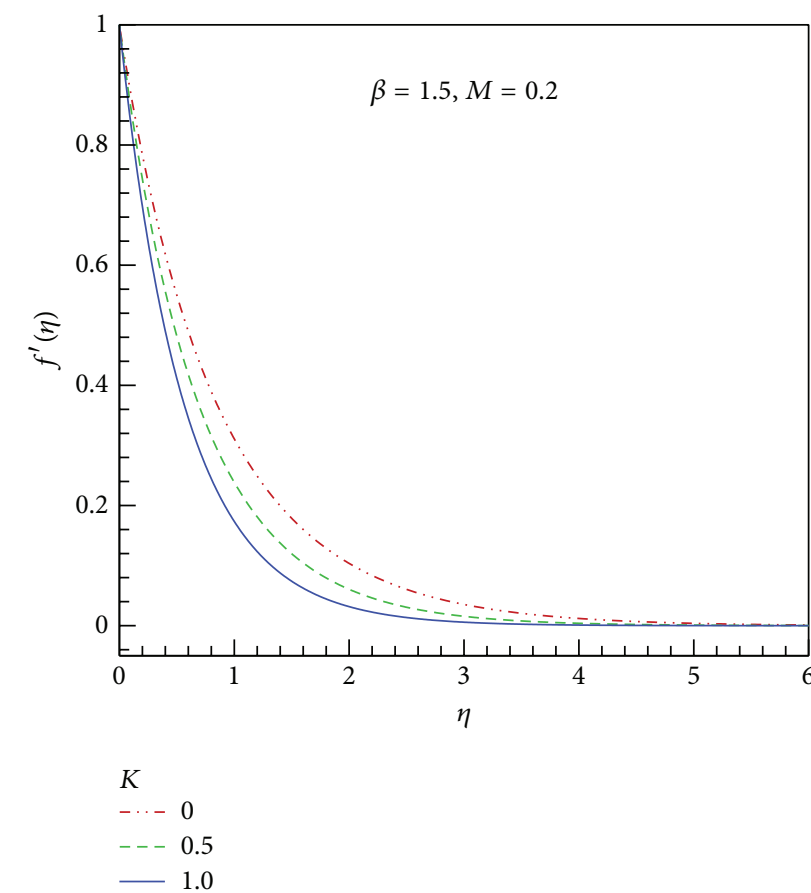

FIGURE 1: Effect of wall mass transfer parameter on dimensionless velocity for $\beta=1.5, M=0.2$.

Figure 1 is displayed for the influence of $K$. It is observed that the dimensionless velocity and associated boundary layer thickness decrease with an increase in $K$. Figures 2 and 3 are given for the velocity profile against $\eta$ in order to show the influences of parameters $M, \beta$, respectively. Figure 2 exhibits the effect of magnetic parameter on the dimensionless velocity. It is observed that the velocity profile of the fluid is significantly reduced with increasing values of $M$. Physically an increase in magnetic parameter $M$ results in a strong reduction in dimensionless velocity $f^{\prime}$. This is due to the fact that magnetic field introduces a retarding body force which acts transverse to the direction of the applied magnetic field. This body force, known as the Lorentz force, decelerates the boundary layer flow and thickens the momentum boundary layer and hence induces an increase in the absolute value of the velocity gradient at the surface as

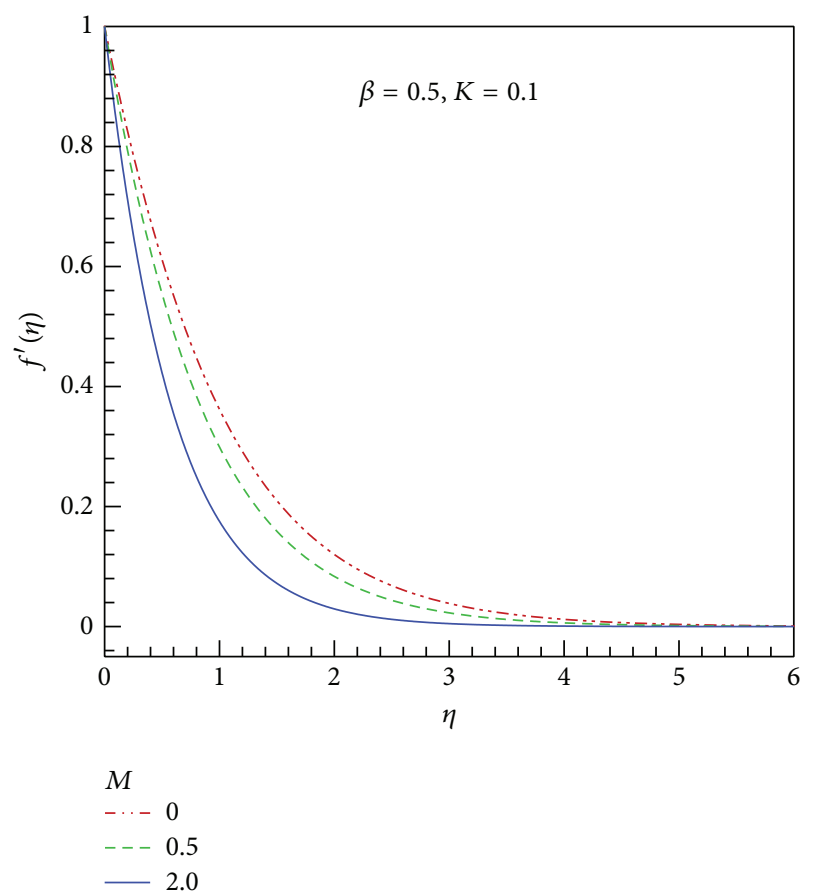

FIGURE 2: Effect of magnetic parameter on dimensionless velocity for $\beta=0.5, K=0.1$.

shown in Table 2. Figure 3 is plotted to show the influence of $\beta$. The dimensionless velocity decreases with an increase in $\beta$ and it is also seen that the hydrodynamics boundary layer thickness is higher for small value of $\beta$.

\section{Concluding Remarks}

In this study, we have successfully applied the optimal homotopy asymptotic method for MHD flow over a nonlinear porous stretching sheet. Both numerical and approximate analytical results are obtained for the problem. The results are presented in tabular and graphical forms for different controlling parameters. It was found that OHAM results are closer to numerical results. The solution obtained using OHAM is also consistent with solution obtained using a numerical method for variation in $\beta, M$, and $K$. 


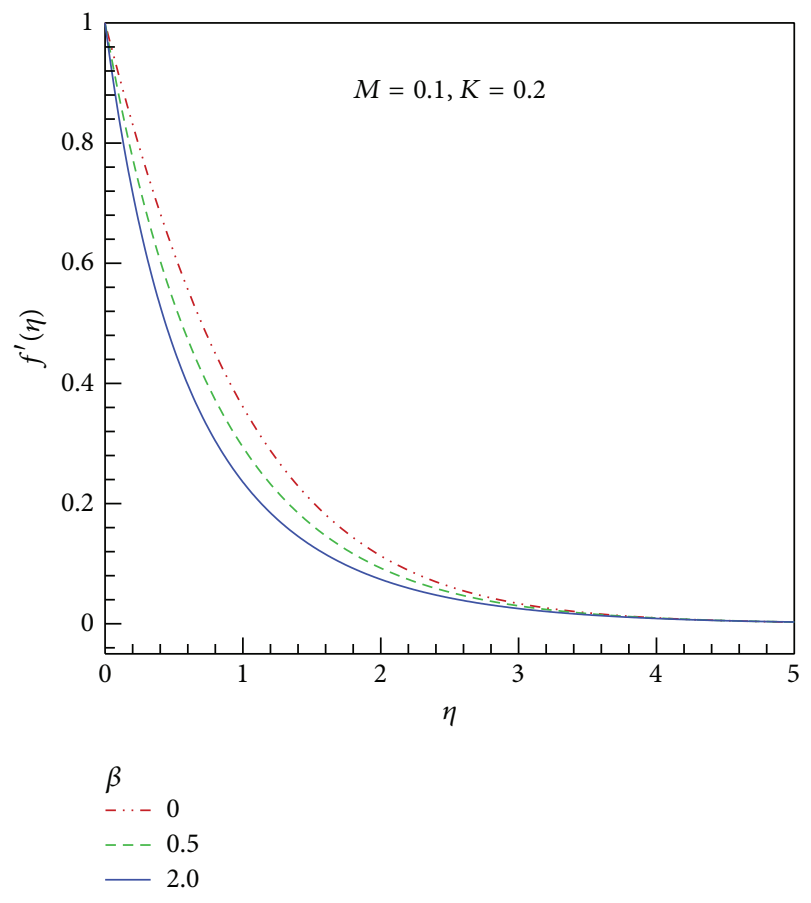

FIGURE 3: Effect of $\beta$ parameter on dimensionless velocity for $K=$ $0.2, M=0.1$.

\section{Competing Interests}

There is no conflict of interests with any person/organization upon the acceptance of this paper.

\section{Acknowledgments}

This research is supported by the Thailand Research Fund under the TRG Research Scholar Grant no. TRG5780016.

\section{References}

[1] A. H. Nayfeh, Introduction to Perturbation Techniques, John Wiley \& Sons, 1979.

[2] R. H. Rand and D. Armbruster, Perturbation Methods, Bifurcation Theory and Computer Algebraic, Springer, Berlin, Germany, 1987.

[3] M. E. Ali, "On thermal boundary layer on a power-law stretched surface with suction or injection," International Journal of Heat and Fluid Flow, vol. 16, no. 4, pp. 280-290, 1995.

[4] J. E. Magyari and B. Keller, "Exact solutions for self-similar boundary-layer flows induced by permeable stretching walls," European Journal of Mechanics-B/Fluids, vol. 19, no. 1, pp. 109122,2000

[5] E. Magyari, I. Pop, and B. Keller, “The 'missing' similarity boundary-layer flow over a moving plane surface," Zeitschrift fur Angewandte Mathematik und Physik, vol. 53, no. 5, pp. 782-793, 2002.

[6] M. Zakaria, "Magnetohydrodynamic viscoelastic boundary layer flow past a stretching plate and heat transfer," Applied Mathematics and Computation, vol. 155, no. 1, pp. 165-177, 2004.
[7] H. S. Takhar, A. A. Raptis, and C. P. Perdikis, "MHD asymmetric flow past a semi-infinite moving plate," Acta Mechanica, vol. 65, no. 1, pp. 287-290, 1987.

[8] K. B. Pavlov, "Oscillatory magnetohydrodynamic flows of viscoplastic media in plane ducts," Magnetohydrodynamics, vol. 9, no. 2, pp. 197-199, 1973.

[9] I. Pop, M. Kumari, and G. Nath, "Conjugate MHD flow past a flat plate," Acta Mechanica, vol. 106, no. 3-4, pp. 215-220, 1994.

[10] A. J. Chamkha, "MHD-free convection from a vertical plate embedded in a thermally stratified porous medium with Hall effects," Applied Mathematical Modelling, vol. 21, no. 10, pp. 603609, 1997.

[11] H. S. Takhar, A. J. Chamkha, and G. Nath, "Flow and mass transfer on a stretching sheet with a magnetic field and chemically reactive species," International Journal of Engineering Science, vol. 38, no. 12, pp. 1303-1314, 2000.

[12] P. D. McCormack and L. Crane, Physics of Fluid Dynamics, Academic Press, New York, NY, USA, 1973.

[13] B. C. Sakiadis, "Boundary-layer behavior on continuous solid surfaces: I. Boundary-layer equations for two-dimensional and axisymmetric flow," AIChE Journal, vol. 7, no. 1, pp. 26-28, 1961.

[14] B. C. Sakiadis, "Boundary-layer behavior on continuous solid surface: II. Boundary-layer equations for two-dimensional and axisymmetric flow," AIChE Journal, vol. 7, pp. 221-225, 1961.

[15] F. K. Tsou, E. M. Sparrow, and R. J. Goldstein, "Flow and heat transfer in the boundary layer on a continuous moving surface," International Journal of Heat and Mass Transfer, vol. 10, no. 2, pp. 219-235, 1967.

[16] M. E. Ali, "Heat transfer characteristics of a continuous stretching surface," Warme-Und Stoffubertragung, vol. 29, no. 4, pp. 227-234, 1994.

[17] E. Magyari and B. Keller, "Heat and mass transfer in the boundary layers on an exponentially stretching continuous surface," Journal of Physics D: Applied Physics, vol. 32, no. 5, pp. $577-585,1999$.

[18] P. D. Ariel, "Computation of flow of viscoelastic fluids by parameter differentiation," International Journal for Numerical Methods in Fluids, vol. 15, no. 11, pp. 1295-1312, 1992.

[19] P. D. Ariel, "On the second solution of flow of viscoelastic fluid over a stretching sheet," Quarterly of Applied Mathematics, vol. 33, no. 4, pp. 629-632, 1995.

[20] B. S. Dandapat and A. S. Gupta, "Flow and heat transfer in a viscoelastic fluid over a stretching sheet," International Journal of Non-Linear Mechanics, vol. 24, no. 3, pp. 215-219, 1989.

[21] M. Fathizadeh, M. Madani, Y. Khan, N. Faraz, A. Yildirim, and S. Tutkun, "An effective modification of the homotopy perturbation method for MHD viscous flow over a stretching sheet," Journal of King Saud University-Science, vol. 25, no. 2, pp. 107-113, 2013.

[22] P. D. Ariel, "MHD flow of a viscoelastic fluid past a stretching sheet with suction," Acta Mechanica, vol. 105, no. 1-4, pp. 49-56, 1994.

[23] Y. Khan, M. A. Abdou, N. Faraz, A. Yildirim, and Q. Wu, "Numerical solution of MHD flow over a nonlinear porous stretching sheet," Iranian Journal of Chemistry and Chemical Engineering, vol. 31, no. 3, pp. 125-132, 2012.

[24] T. C. Chiam, "Hydromagnetic flow over a surface stretching with a power-law velocity," International Journal of Engineering Science, vol. 33, no. 3, pp. 429-435, 1995.

[25] K. B. Pavlov, "Magnetohydrodynamic flow of an incompressible viscous fluid caused by deformation of a plane surface," Magnetohydrodynamics, vol. 10, no. 4, pp. 507-510, 1974. 
[26] V. Marinca, N. Herişanu, T. Dordea, and G. Madescu, "A new analytical approach to nonlinear vibration of an electrical machine," Proceedings of the Romanian Academy Series A, vol. 9, no. 3, pp. 229-236, 2008.

[27] V. Marinca and N. Herişanu, "Application of Optimal Homotopy Asymptotic Method for solving nonlinear equations arising in heat transfer," International Communications in Heat and Mass Transfer, vol. 35, no. 6, pp. 710-715, 2008.

[28] V. Marinca and N. Herişanu, "Determination of periodic solutions for the motion of a particle on a rotating parabola by means of the optimal homotopy asymptotic method," Journal of Sound and Vibration, vol. 329, no. 9, pp. 1450-1459, 2010. 


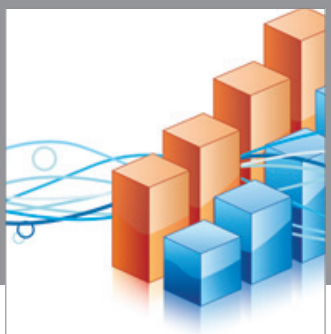

Advances in

Operations Research

vatem alat4

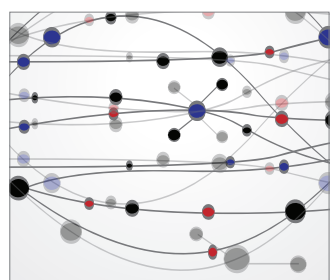

\section{The Scientific} World Journal
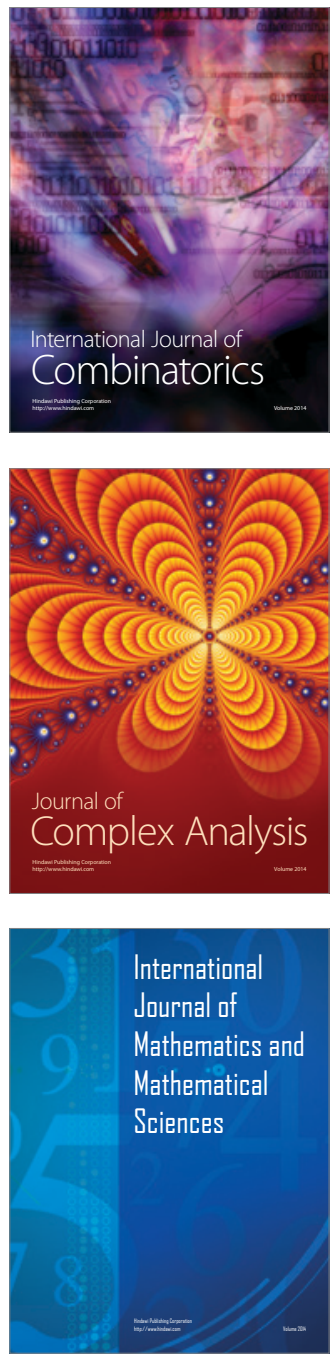
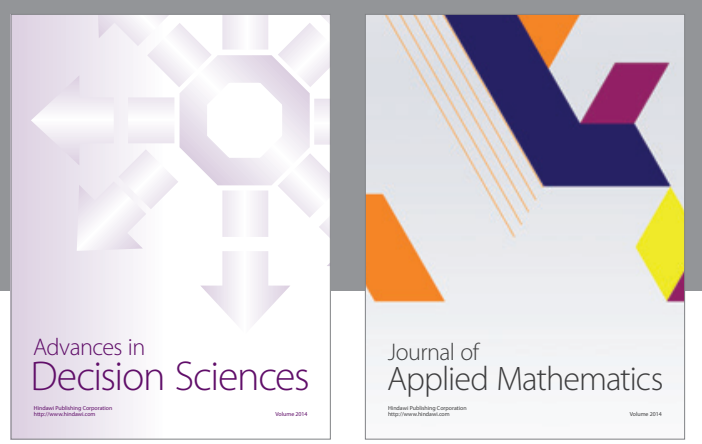

Algebra

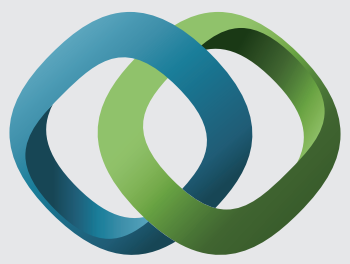

\section{Hindawi}

Submit your manuscripts at

http://www.hindawi.com
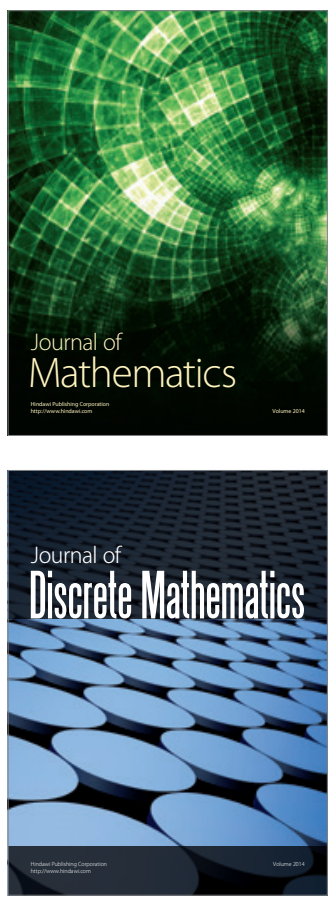

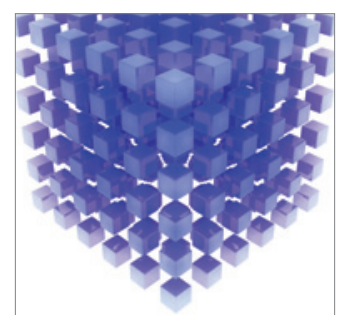

Mathematical Problems in Engineering
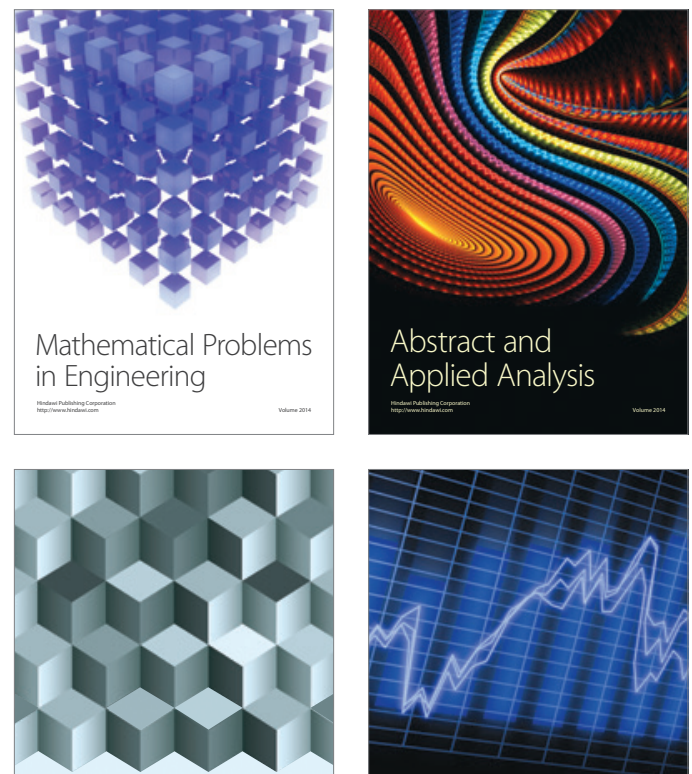

Journal of

Function Spaces

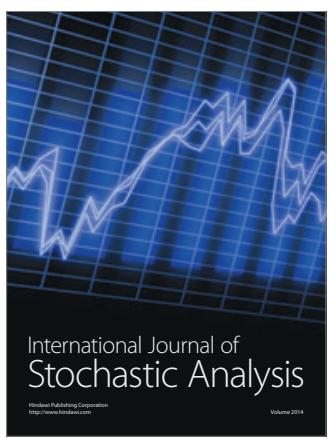

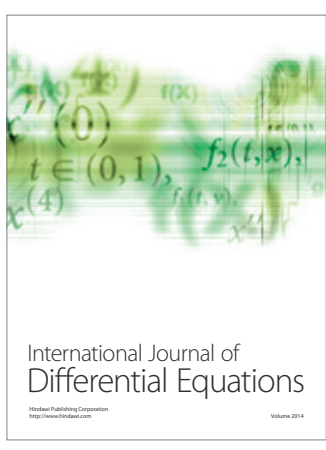
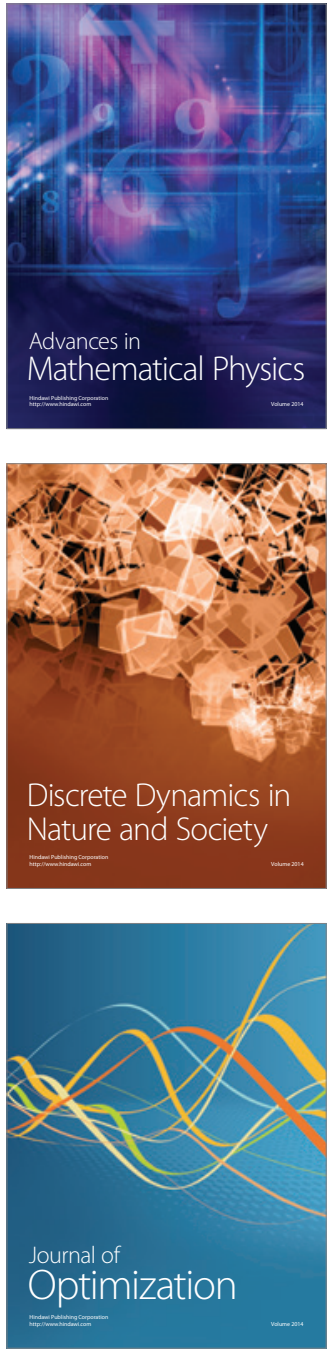